\title{
Metastatic Pulmonary Calcification An Uncommon Clinical Condition in End-Stage Renal Disease
}

\author{
Gaurav Patel MD, Andre Yepes-Hurtado MD, Isham Huizar MD
}

\begin{abstract}
Patients with end-stage renal diseases have frequent pulmonary complications related to fluid overload, infection, and associated cardiovascular diseases. We report a 73-year-old man with renal failure on peritoneal dialysis who had elevated serum calcium, phosphorous, and parathyroid hormone levels. He had progressive dyspnea and an abnormal chest $x$-ray. A high resolution computed tomography scan revealed calcified nodules, large cavities with heavy calcification in the upper zones, calcifications in the tracheobronchial tree, and calcified vessels. This patient had metastatic pulmonary calcification and possibly dystrophic calcification due to renal failure which probably contributed to his pulmonary symptoms.
\end{abstract}

Key words: end-stage renal disease, pulmonary calcifications, high resolution CT

\section{INTRODUCTION}

Metastatic pulmonary calcification (MPC) is an unusual condition in which the deposition of calcium salts occurs in normal alveoli, interstitium, and bronchovascular tissue. ${ }^{1,2,3}$ MPC occurs in end-stage renal disease (ESRD) and other disorders, such as hyperparathyroidism and diffuse skeletal malignancy, associated with hypercalcemia. Dystrophic calcification occurs in diseases associated with lung injury, inflammation, and necrosis, including granulomatous infections, viral infections, sarcoidosis, infraction, and pneumoconiosis. ${ }^{4,5,6}$ We describe a case of MPC in a patient with ESRD treated by peritoneal dialysis and presenting with progressive dyspnea.

Corresponding author: Gaurav Patel MD

Contact Information: gaurav.patel@ttuhsc.edu DOI: 10.12746/swrccc2014.0205.059

\section{Case Presenttion}

A 73-year-old man was referred to the pulmonary clinic with a seven month history of gradually progressive dyspnea (NYHA grade III dyspnea) and persistent bilateral infiltrates on chest x-ray. He was diagnosed with ESRD three years earlier and was undergoing regular peritoneal dialysis. He had a 25-pack-year smoking history but had quit smoking 23 years ago. He had no prior history of tuberculosis or exposure to tuberculosis. His medications were sevelamer, vitamin D3, ferrous sulfate, midodrine, aspirin, and simvastatin. He denied constitutional symptoms, recent weight loss, hemoptysis, chronic cough, and sputum production. He worked as a farmer for more than 20 years and was now retired. On clinical examination, he appeared his stated age, was overweight, and was using supplemental oxygen. He had grade I peripheral edema but no jugular venous distention. Auscultation of the chest revealed rales in both upper and middle zones of lungs and normal 
heart sounds without any murmurs. Laboratory tests revealed hemoglobin $10 \mathrm{~g} / \mathrm{dl}$, blood urea nitrogen 49 $\mathrm{mg} / \mathrm{dl}$, and serum creatinine $10.9 \mathrm{mg} / \mathrm{dl}$, serum calcium $11.1 \mathrm{mg} / \mathrm{dl}$, serum phosphorus $5.3 \mathrm{mg} / \mathrm{dl}$, and an elevated parathyroid hormone (PTH) level at 124 $\mathrm{pg} / \mathrm{ml}$ (normal 15-65 pg/ml). The calcium and phosphorus product (Ca*Po4) was $58.8 \mathrm{mg}^{2} / \mathrm{dl}^{2}$ (desirable range $<55 \mathrm{mg}^{2} / \mathrm{dl}^{2}$ ).

His chest $\mathrm{x}$-ray revealed multifocal bilateral infiltrates. In the anterior lower right upper lobe there was a dense opacity with an approximate size of $6 \mathrm{~cm}$ by $5 \mathrm{~cm}$ and in the left upper lobe there were multiple densities of various diameters from subcentimeter to $1.5 \mathrm{~cm}$ in size (Figures $1 \mathrm{~A}$ and $\mathrm{B}$ ). A high resolution computed tomography (HRCT) scan showed irregular nodular densities scattered in both upper lobes and in the right middle lobe (the largest lesion measured 2 $\mathrm{cm} \times 1.3 \mathrm{~cm}$ ) (Figures $2 \mathrm{~A}$ and $\mathrm{B}$ and Figure $3 \mathrm{~A}$ and $B)$. Calcific densities were present in the cavitary le- sions; the tracheobronchial tree and vessels had calcifications. The HRCT also revealed emphysematous and bronchiectatic changes. There were no mediastinal lymph nodes or pleural effusions.

The patient's pulmonary function test revealed moderate airflow obstruction and air trapping with a FEV1 1.9 L (63\% predicted), FVC 3.2 L (83\% predicted), FEV1/FVC ratio 0.59 , TLC $7.3 \mathrm{~L}$ (117\% predicted), RV 4.0L (153\% predicted), and DLCO 40\% predicted. His severe decrease in DLCO could also reflect underlying pulmonary vascular disease.

The constellation of progressive symptoms, distinct radiological finding, chronic renal failure, and secondary hyperparathyroidism suggested that the patient had MPC. However, the dense calcifications in some areas also suggest that he had dystrophic calcifications associated with prior lung injury or with underlying bullous lung disease.
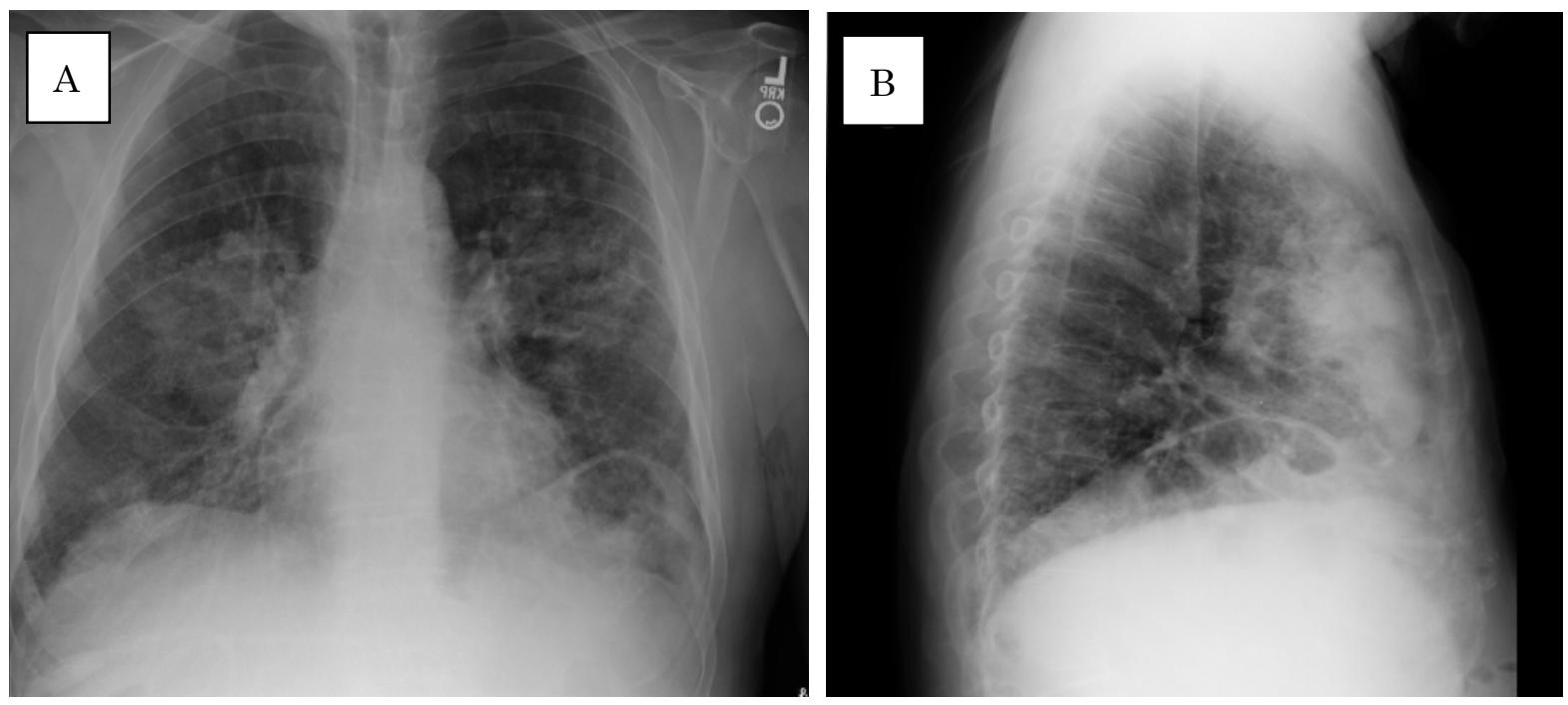

Figure 1. Chest $\mathrm{x}$-rays (A-postero-anterior view; B- lateral view) reveal diffuse bilateral pulmonary opacities in upper and anterior regions of the lung parenchyma. 

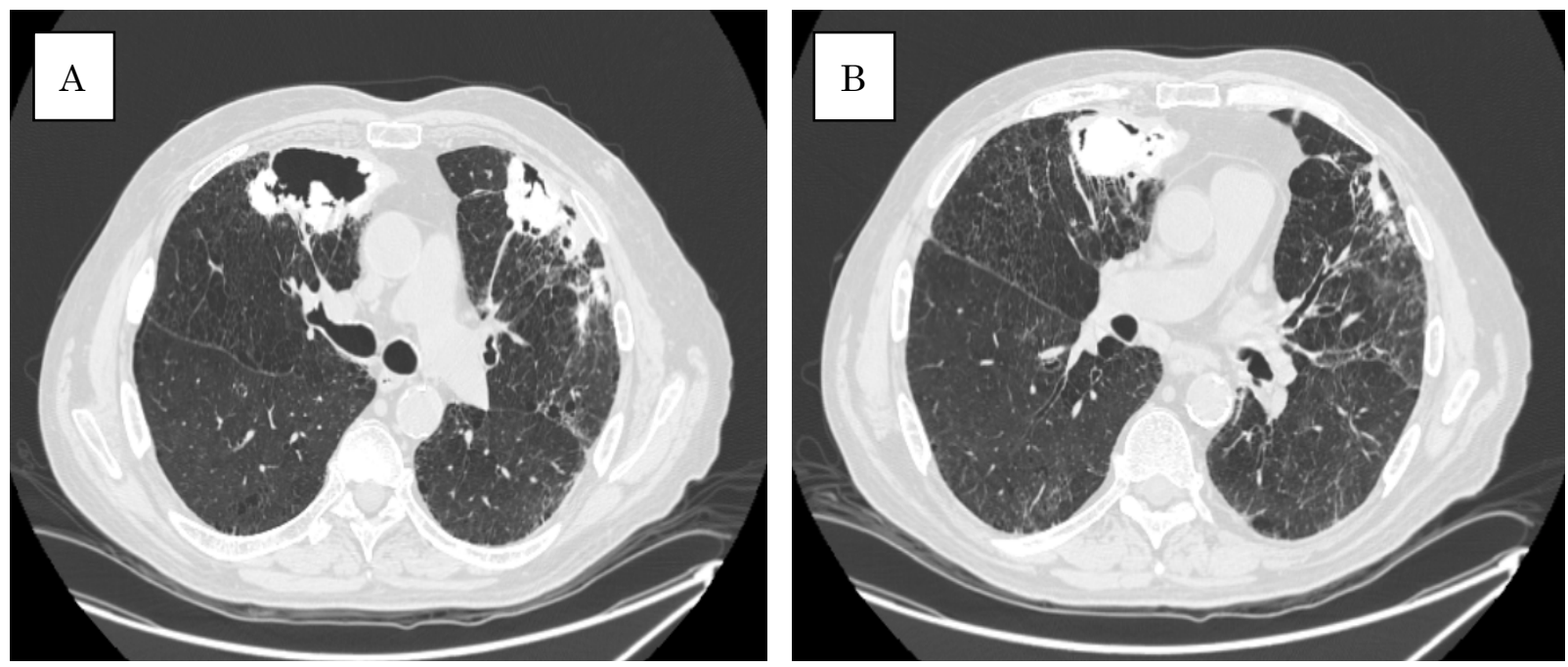

Figure 2. High resolution computed tomography scans (HRCT) (A and B) show subpleural cavitary lesions with large, irregular peripheral dystrophic calcifications in the anterior regions of both upper lobes lower. Bullous changes, peribronchial thickening, and bronchiectatic changes are also noted. Additionally, there are irregular nodular densities scattered bilaterally in the upper lungs.
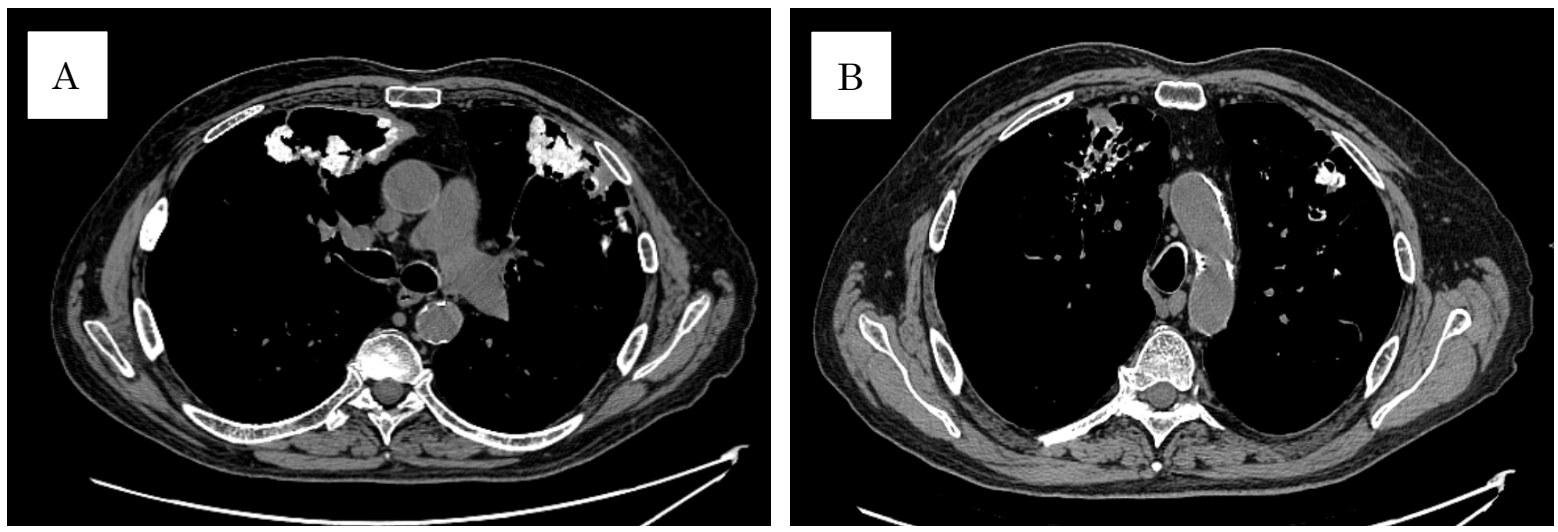

Figure 3. The mediastinal windows of $\operatorname{HRCT}(\mathrm{A}$ and $\mathrm{B})$ show pleural based mass-like densities with cavitation in the anterior region of both upper lobes. Prominent calcifications are noted inside and around wall of cavities. Calcifications can be seen in the wall of the aorta and trachea.

\section{Discussion}

The exact mechanism(s) of lung calcification with or without ossification is not known. ${ }^{6}$ It develops as a result of abnormalities in calcium and phosphate metabolism, alkaline phosphatase activity, and local physicochemical conditions, such as changes in $\mathrm{pH}^{6,7}$
The development of MPC does not closely correlate with serum calcium levels, phosphate levels, parathyroid hormone levels, or with the duration of hemodialysis. ${ }^{8}$ Calcium phosphate is the principal mineral salt of ESRD-associated metastatic calcification, and the composition of MPC is analogous to normal bone. 
ESRD patients on dialysis have four conditions that predispose them to the development of pulmonary calcification. First, acidosis leaches calcium and phosphate from bone. Second, failure to convert 25-hydroxyvitamin $D$ to 1,25 -dihydroxyvitamin $D$ by the kidneys leads to a low calcium balance and increased parathyroid hormone secretion. Third, intermittent alkalosis often accompanies bicarbonate hemodialysis and increases soft tissue precipitation of calcium salts. Finally, the decreased glomerular filtration of phosphate may contribute to an elevated serum calcium-phosphate product. In uremic patients, elevated serum phosphate levels correlate with vascular calcification. ${ }^{6}$ This calcification develops over time and is often detected incidentally. Some patients may develop restrictive physiology with a low diffusion capacity and respiratory failure. ${ }^{6,7}$ Severe calcification may cause interstitial fibrosis. Chest radiography provides the initial evaluation of MPC but is not very sensitive. ${ }^{9}$ Chest radiographs are often normal, and when abnormal reveal ill-defined nodules in upper lobes which may suggest prominent vessels or infection. ${ }^{1}$ Computed tomography (CT) is a highly sensitive imaging technique for the diagnosis of MPC. ${ }^{1,4}$ These scans show coarse reticular opacities, thickened reticulonodular infiltrates, and bone density lesions. ${ }^{6}$ In addition, patchy consolidation, ground glass opacities, dense lobar consolidation, and calcification of chest wall vessels and the tracheobronchial tree also occur. ${ }^{1,10}$ High resolution CT (HRCT) scans are very useful in patients with small nodules. Bone scintigraphy with Tc-99m-MDP with uptake of the radioactive tracer into the lung parenchyma or HRCT using mediastinal windows to evaluate the lung parenchyma can identify MPC with a high degree of certainty and eliminate the need for additional evaluation. ${ }^{13,14}$

At autopsy, MPC is found in $60-75 \%$ patients with end-stage renal disease. ${ }^{3}$ The Von Kossa stain is more sensitive than the standard hematoxylin and eosin stain in detecting calcium in tissues. ${ }^{11}$ Electron microscopy is even more sensitive and can show specific intracellular and extracellular sites of calcification. ${ }^{12}$ The differential diagnosis of pulmonary calcifications includes granulomatous infections (Histoplasma cap- sulatum, Coccidioides immitis and Mycobacterium tuberculosis), viral infections (Varicella), parasitic infections (Paragonimus westermani), Pneumocystis jiroveci, silicosis, metastatic malignancy, and alveolar microlithiasis. The presence of pulmonary and vascular calcifications is considered characteristic of MPC. ${ }^{1}$ Our patient had calcifications in the lungs, the tracheobronchial tree, and chest wall vessels typical for MPC. He had increasing dyspnea probably secondary to both COPD and extensive calcification in the lung parenchyma. No definitive treatment is available. Steroids, calcium-binding drugs, and low calcium diets have no proven benefits; the therapeutic effects of bisphosphonates remain uncertain. ${ }^{6}$ Patients are usually treated with phosphate binders. ${ }^{9}$ In advanced cases of secondary hyperparathyroidism, parathyroidectomy can be done.

In summary, MPC is relatively common at autopsy in patients with ESRD, but clinical manifestations are infrequent. HRCT scans and bone scintigraphy provide the best demonstration of calcification in the lungs, the tracheobronchial tree, and vessels. Detection of these findings can make this diagnosis and lead to additional pulmonary evaluation and possible changes in management.

\section{KeYPOINTS}

1. Patients with chronic renal failure can develop calcium deposition in normal lung tissue, including alveolar epithelium, alveolar capillaries, bronchial walls, and pulmonary arterioles.

2. This occurs more frequently in the upper lung zones; plain chest x-rays are often normal.

3. Bone scintigraphy and computed tomography can provide a diagnosis without the need for biopsy.

4. These patients often have no symptoms but can have progressive dyspnea and restrictive physiology. 
Author Affiliation: Gaurav Patel and Andre Yepes-Hurtado are fellows in the pulmonary and critical care division at Texas Tech University Health Science Center Lubbock, TX. Isham Huizar is a faculty member in the pulmonary and critical care division at TTUHSC Lubbock, TX.

Received: 10/15/2013

Accepted: 12/10/2013

Reviewers: Eman Attaya MD, Vaqar Ahmed MD

Published electronically: 01/15/2014

Conflict of Interest Disclosures: None

\section{REFERENCES}

1. Hartman TE, Muller NL, Primack SL et al (1994) Metastatic pulmonary calcification in patient with hypercalcemia: findings on chest radiographs and CT scans. AJR Am J Roentgenol 1994; 162:233-238.

2. Kang EH, Kim ES, Kim CH, Ham SY, Oh YW. Atypical radiological manifestation of pulmonary metastatic calcification. Korean J Radiol 2008; 9:186-189.

3. Cogner JD, Hammond WS, Alfrey AC, Contiguglia SR, Stanford RE, Huffer WE. Pulmonary calcification in chronic dialysis patients: clinical and pathologic studies. Ann Intern Med 1975; 83(3):330-3363.

4. Thurley PD, Duerden R, Roe S, Pointon K. Rapidly progressive metastatic pulmonary calcification: evolution of changes on CT. Br J Radiol 2009; 82:e155-e159.

5. Kuhlman FE, Ren H, Hutchins GM, Fishman EK. Fulminant pulmonary calcification complicating renal transplantation: CT demonstration. Radiology 1989; 73:459-460.

6. Chan ED, Morales DV, Welsh CH, McDermott MT, Schwarz MI. Calcium deposition with or without bone formation in the lung. Am J Respir Crit Care Med 2002; 165:1654-1669.

7. Madhusudhan KS, Shad PS, Sharma S, Goel A, Mahajan H. Metastatic pulmonary calcification in chronic renal failure. Int Urol Nephrol 2012; 44:1285-1287.

8. Sanders C, Frank MS, Rostand SG, Rutsky EA, Barnes GT, Fraser RG. Metastatic calcification of the heart and lungs in endstage renal disease: detection and quantification by dual-energy digital chest radiography. AJR Am J Roentgenol 1987; 149:881887.

9. Rastogi S, Boyars M, Eltorky M, Taneja S, Rouan GW. Metastatic pulmonary calcification in a patient with end stage renal disease on hemodialysis: a common complication but a rare clini- cal diagnosis. Johns Hopkins Adv Study Med 2006; 6:82-85. 10. Yasuo M, Tanabe T, Komatsu Y, et al. Progressive pulmonary calcification after successful renal transplantation. Intern Med 2008; 47:161-164.

11. Nothcutt AD, Tio FO, Chamblin SA, Britton HA. Massive metastatic pulmonary calcification in an infant with aleukemic monocytic leukemia. Pediatr Pathol 1985; 4:219-229.

12. Ghadially FN. As you like it. Part 3. A critique and historical review of calcification as seen with the electron microscope. Ultrastruct Pathol 2001; 25:243-267.

13. Hochhegger B, Marchiori E, Soaressouza Jr. A, Palermo L. MRI and CT findings of metastatic pulmonary calcification. $\mathrm{Br} J$ Radiol 2012; 85:e69-e72.

14. Thurley P, Duerden R, Roe S, Pointon K. (2009) Rapidly progressive metastatic pulmonary calcification: evolution of changes on CT. Br J Radiol 2009; 82: e155-e159. 